\title{
Exact Controllability of Galerkin's Approximations for the Oldroyd Fluid System
}

\author{
A. O. Marinho, A. T. Lourêdo, and M. Milla Miranda
}

\begin{abstract}
In this paper we investigate the model of Oldroyd Fluid in a bounded smooth region of $R n(n=2,3)$ with control supported in a small subset of this domain. Under suitable assumptions on the Galerkin basis, we introduce Galerkin's approximations for the Oldroyd fluid system. Using the Hilbert Uniqueness Method in combination with the Schauder's fixed point, we prove the exact controllability for this finite-dimensional system.
\end{abstract}

Index Terms-Exact controllability, oldroyd fluid, Galerkin'S approximations.

\section{INTRODUCTION}

The focus of this paper is the study of the exact controllability of Galerkin's Approximations for the equation of the Oldroyd fluid. This model corresponding to an incompressible fluid, which is described by the following system of partial differential equations.

$$
\begin{aligned}
& \frac{\partial u}{\partial t}+u . \nabla u+\nabla p=\operatorname{div}(\sigma)+F(x, t), \\
& x \in \Omega, t>0, \operatorname{div}(u)=0, x \in \Omega, t>0
\end{aligned}
$$

With appropriate initial and boundary conditions. Here $\sigma=$ $\left(\sigma_{i k}\right)$ denotes the stress tensor with $\operatorname{tr} \sigma=0, u$ represents the velocity vector, $p$ is the pressure in the fluid and $F$ is the external force. In fact, the stress tensor $\sigma$ plays a special role, because the introduction of $\sigma$ in (1) has the purpose of letting us consider reactions arising in the fluid during its motion. By establishing (Hookes's Law) the connection between $\sigma$ and the tensor of deformation velocities $D=\left(D_{i k}\right)=\frac{1}{2}\left(u_{i x_{k}}+u_{k x_{i}}\right)$, and their derivatives, we thus establish the type of fluid. Such relation between $\sigma$ and $D$ is called a defining or rheological equation or an equation of state (see [1]). From Newton's law, we set

$$
\sigma=2 v D
$$

where is $v$ the kinematic coefficient of viscosity, such fluid is called of Newtonian Fluid. Substituting (2) in (1) we obtain the equations of motion of Newtonian fluid, which is called Navies-Stakes equations.

Over the last century and half, the model of a Newtonian fluid has been the basic model of a viscous incompressible fluid. It describes flows of moderate velocities of the majority

Manuscript received December 24, 2013; revised February 28, 2014

Aldo Trajano Lourêdo is with the mathematics Department at UEPB. Brazil (e-mail: aldotl@cct.uepb.edu.br). of viscous incompressible fluids encountered in practice. However, even eelier in the middle nineteenth century it was known that there exists viscous incompressible fluid not subject to the Newtonian equation (2). That is, it has a complex microstructure such as biological fluids, suspensions and liquid crystals, which are used in the current industrial process and shows (non-linear) viscoelastic behavior that cannot be described by the classical linear viscous Newtonian models. The first models of such fluids, were proposed in the nineteenth century by Maxwell [2] and [3], Kelvin [4], Voigt [5] and [6]. In the mid twentieth century Oldroyd extended such models [7]-[8].

The model of Oldroyd fluid (see [9], [7], [10]) can predict the stress relaxation as well as the retardation of deformation. Due to this, it has become popular for describing polymer suspension. In order to model the behavior of a dilute polymer solution in a Newtonian solvent, the extra stress tensor is often split in two components: a viscoelastic one and a purely viscous one. So the Oldroyd fluids of order one as it is known in the Russian literature (see [7], [11], [12], [13] and [14]) are described by defining relation.

$$
\left(1+\lambda \frac{\partial}{\partial t}\right) \sigma=2 v\left(1+k v^{-1} \frac{\partial}{\partial t}\right) D
$$

where $\lambda, v, k$ are positive constants with $(v-k \lambda-1)>0$. Here $v$ denotes the kinematic viscosity, $\lambda$ is the relaxation time, and $\mathrm{k}$ represents the retardation time. In the form of an integral equation, we write the above defining relation as.

$$
\begin{aligned}
& \sigma(x, t)=2 k \lambda^{-1} D(x, t)+ \\
& 2 k \lambda^{-1}\left(v-k \lambda^{-1}\right) \int_{0}^{t} e^{\frac{-(t-\xi)}{\lambda}} D(x, t) d \xi
\end{aligned}
$$

were $\sigma(x, 0)=0=D(x, 0)$.

Thus the equation of motion of the Oldroyd fluids of order one can be described most naturally by the system of integro-differential equations.

$$
\begin{gathered}
\frac{\partial u}{\partial t}+(u \cdot \nabla) u-\mu \Delta u- \\
-\int_{0}^{t} \beta(t-\xi) \Delta u(x, \xi) d \xi+\nabla p=f, x \in \Omega, t>0,(5)
\end{gathered}
$$

And the incompressible condition 


$$
\operatorname{div}(u)=0, x \in \Omega, t>0
$$

With initial and boundary conditions

$$
u(x, 0)=u_{0}, x \in \Omega, \text { and } u(x, t)=0 x \in \Gamma, t \geq 0 .
$$

Here, $\Omega$ is an open bounded connected set of $\mathbb{R}^{n}$ with smooth boundary $\Gamma, \quad \mu=k \lambda^{-1} \quad$ and kernel $g(t)=\gamma e^{-\delta t}$, where $\gamma=\lambda^{-1}\left(v-k \lambda^{-1}\right)$ and $\delta=\lambda-1$. For details of the physical background and its mathematical modeling, see [7], [9], [10] and [11].

As in Temam [15] let us denote $\operatorname{Hm}(\Omega)$ the standard Hilbert-Sobolev space and by $\|.\| \mathrm{m}$ the norm defined on it. When $m=0$, we call $\mathrm{H0}(\Omega)$ as the space of square integrable functions $L^{2}(\Omega)$ with the usual norm $\mid$. | and inner product (., .). Further, let $H_{0}^{1}(\Omega)$ be the completion of $C_{0}^{\infty}(\Omega)$ with respect to $H_{0}^{1}(\Omega)$-norm. We also use the following function spaces for the vector valued functions.

Let us consider the spaces:

$$
V:=\left\{\varphi \in\left(C_{0}^{\infty}(\Omega)\right)^{n}: \operatorname{div}(\varphi)=0 \text { in } \Omega, t>0\right\}
$$

$H:=$ the closure of $V$ in $\left(L^{2}(\Omega)\right)^{n}$ and $V:=$ the closure of $\mathrm{V}$ in $\left(H_{0}^{1}(\Omega)\right)^{n}$.

The spaces of vector functions are indicated by boldface, for instance, $\quad H_{0}^{1}(\Omega)=\left(H_{0}^{1}(\Omega)\right)^{n}$ $L^{2}(\Omega)=\left(L^{2}(\Omega)\right)^{n}$. The inner products on $H_{0}^{1}(\Omega)$ and $L^{2}(\Omega)$ are denoted by

$$
((\phi, \omega))=\sum_{i=1}^{n}\left(\nabla \phi, \nabla \omega_{i}\right) \quad \text { and } \quad(\varphi, \omega)=\sum_{i=1}^{n}\left(\varphi_{i}, \omega_{i}\right)
$$

respectively and norm by

$$
\|\varphi\|=\left(\sum_{i=1}^{n}\left|\nabla \varphi_{i}\right|^{2}\right)^{\frac{1}{2}} \text { and }|\phi|=\left(\sum_{i=1}^{n}\left|\phi_{i}\right|^{2}\right)^{\frac{1}{2}}
$$

Respectively:

Note that under some smoothness assumptions on the boundary $\Gamma$, it is possible to characterize $H$ and $V$ as

$$
\begin{aligned}
& H=\left\{u \in \boldsymbol{L}^{2}(\Omega) ; \operatorname{div}(u)=0,\left.u \cdot \eta\right|_{\Gamma}=0\right\} \\
& V=\left\{u \in \boldsymbol{H}_{0}^{1}(\Omega) ; \operatorname{div}(u)=0\right\}
\end{aligned}
$$

See Temam [15]. By Poincaré inequality, it can be shown that the norm of $\boldsymbol{H}_{0}^{1}(\Omega) \quad$ is equivalent to $\boldsymbol{H}^{1}(\Omega)=\left(H^{1}(\Omega)\right)^{n}$ -norm. By $V^{\prime}$ we denote the dual of $V$.

Let $T>0$ a real number. We denote by $Q$ the cylinder $\Omega$ $\times(0, T)$ of $\mathbb{R}^{n+1}$ with lateral boundary $\sum=\Gamma \times(0, T)$. Let be $a \Theta$ non-empty open subset of $\Omega$. By we represent the characteristic functions on $\Theta$. The main focus of this paper is to discuss the exact controllability for system

$$
\mid \begin{aligned}
& \frac{\partial u}{\partial t}-\mu \Delta u+(u \cdot \nabla) u-\int_{0}^{1} g(t-s) \Delta u(s) d s+\nabla p=\mathrm{v} \chi_{\Theta} \text { in } Q \\
& \operatorname{div}(u)=0 \text { in } Q \\
& u=0 \text { on } \sum \\
& u(x, 0)=u_{0}(x) \text { on } \Omega
\end{aligned}
$$

where $\mathcal{u}(x t)=\left(u_{1}(x, t), u_{2}(x, t), u_{3}(x, t)\right)$ is the vector velocity (or state of the system) of fluid evaluated at the point $(x, t), x=\left(x_{1}, x_{2}, x_{3}\right) \in \mathbb{R}^{3}, p=p(x, t)$ is the pressure of the fluid evaluated at the point $(\mathrm{x}, \mathrm{t}), \mu$ represent a constant, $u_{0}(x)$ is the initial velocity and $g(t)=\gamma e^{-\delta t}$, where $\gamma=\lambda^{-1}(v-$ $\left.k \lambda^{-1}\right)$ and $\delta=\lambda^{-1}$. The function $v=v(x, t)$ is the control function distributed in $\mathcal{O}$.

In the context of the Navier-Stokes System, there are results of the local exact controllability to uncontrolled trajectories obtained in Fursikovlmanuvilov [16], Imanuvilov [17] and Femández-Cara et al., [18]. The global approximate controllability of the 2-D Navier-Stokes equations with slip boundary conditions was obtained by Coron [19]. Combining results on global approximate controllability and local controllability, the global exact controllability for the Navier-Stokes system on a 2-D manifold was analyzed in Coron-Fursikov [20]. In [21] and [22], Lions and Zuazua introduced the finite-dimensional Galerkin's approximations for the Navier-Stokes system, and they proved that these Galerkin's approximations are exactly controllable and Araruna, Chaves-Silva and Rojas Medar [23] proved exact controllability of Galerkin's approximations of micropolar fluids. Optimal control problems associated with the Navier-Stokes equations also have a wide and important range in applications. This issue has been studied, for instance, by Fursikov in [24] and by Gunzburger and Hou in [25] and [26]. Concerning control results for the micropolar fluids, Femández-Cara and Guerrero in [27].

Our paper is organized as follows: In Section II, we state the basic notations. In Section III, we in traduce Galerkin's approximations for (1.1) and, for this finite dimensional system, we establish the exact controllability result. The proof is based on the Hilbert Uniqueness Method introduced by Lions (see, for instance, [28]) to study the exact controllability of linear systems and a fixed point technique.

\section{GALERKIN'S APPROXIMATIONS}

We represent the problem (1) in the variational formulation for all $\varphi \in V$,

$$
\mid \begin{aligned}
& (u, \varphi)+\mu a(u, \varphi)+((u \cdot \nabla) u, \varphi)+a(g * u, \varphi)=\left(\mathrm{v} \chi_{\odot}, \varphi\right) \\
& u(x, 0)=u_{0}(x) \text { in } \quad V,
\end{aligned}
$$

For all $\varphi \in \boldsymbol{V}$

$$
a(u, \mathrm{v})=\sum_{i, j=1}^{3} \int \frac{\partial u_{i}}{\partial x_{j}} \frac{\partial \mathrm{v}_{i}}{\partial x_{j}} d x
$$


And $(g * u)(t)=\int_{0}^{t} g(t-s) u(s) d s$

In $V$ we consider a base $\left\{e_{j}\right\}_{j \geq 1}$ such that $\left\{e_{j}\right\}_{j \geq 1}$ are linearly independent in $\boldsymbol{L}^{2}(\Theta)$.

The existence of this basis is guaranteed due to an abstract result proved in [19].

Let $E$ be the finite-dimensional space

$$
E=\operatorname{span}\left[e_{1}, \ldots . ., e_{n}\right]
$$

We introduce the Galerkin's approximations of the variation formulation (9) i.e.

$$
\mid \begin{aligned}
& \left(u_{t}, e\right)+\mu a(u, e)+((u \cdot \nabla) u, e)+a(g * u, e)=\left(\mathrm{v} \chi_{\mathcal{O}}, e\right) \\
& u(x, 0)=u_{0}(x) \text { in } E,
\end{aligned}
$$

For all $e \in E$

System (12) has a unique solution, $u \in C^{0}([0, T] ; F)$ (see Apendix).

We say that (12) is exactly controllable in time $\mathrm{T}>0$ if, given $u_{0}, u^{T} \in E$, there exists a control $\mathrm{v} \in \boldsymbol{L}^{2}(\vartheta \times(0, T))$ such that the solution $u$ of (12) satisfies

$$
u(., T ; \mathrm{v})=u^{T}
$$

We introduce the functional cost given by

$$
J(u)=\frac{1}{2} \int_{\Theta \times(0, T)}|u|^{2} d x d t, u \in \boldsymbol{L}^{2}(\Theta \times(0, T)
$$

$n$ these conditions proof the following result:

Theorem 2.1. Let $T>0$ be given. Then the Galerkins approximations (12) is exactly controllable in the sense of (13). Furthermore, the functional cast of control given in (14) is bounded independently of the nonlinearity.

Proof. later on, to show and make explicit the cost of control can be bounded independently of the non- linearity, we introduce the family of systems

$$
\mid \begin{aligned}
& \frac{\partial u}{\partial t}-\mu \Delta u+(u \cdot \nabla) u-\int_{0}^{1} g(t-s) \Delta u(s) d s+\nabla p=\mathrm{v} \chi_{\Theta} \text { in } Q \\
& \operatorname{div}(u)=0 \text { in } Q \\
& u=0 \text { on } \sum \\
& u(x, 0)=u_{0}(x) \text { on } \Omega
\end{aligned}
$$

where $\alpha \in R$. We will find some estimates independent of $\alpha$.

We prove our main result for system (15). First, we introduce its variation formulation. Namely

$$
\mid \begin{aligned}
& \left(u_{t}, e\right)+\mu a(u, e)+((u \cdot \nabla) u, e)+\alpha(g * u, e)=\left(\mathrm{v} \chi_{\ominus}, e\right) \\
& u(x, 0)=u_{0}(x) \text { in } E,
\end{aligned}
$$

We proceed with this proof in several steps

Step 1 (Linear System). Take a function $h$ such that

$$
h \in L^{2}(0, T ; E)
$$

And we consider the linear system

$$
\mid \begin{aligned}
& \left(u_{t}, e\right)+\mu a(u, e)+((h \cdot \nabla) u, e)+\alpha(g * u, e)=\left(\mathrm{v} \chi_{\odot}, e\right) \\
& u(x, 0)=u_{0}(x) \text { in } E
\end{aligned}
$$

for all $e \in E$

System (18) has a unique solution $u \in C^{0}([0, T] ; E)$, therefore due to the linearity of the problem we can be assume the data null. But, all results are valid as well if the initial data is not zero, that is, $u(0)=u_{0} \in E$.

Let us check that system (18) is exactly controllable in any time $T>0$, in the sense of (13). For this, it suffices to prove that if $\beta \in$ E satisfies

$$
(u(., T ; \mathrm{v}), \beta)=0, \forall \mathrm{v} \in L^{2}(\Theta \times(0, T)) \Rightarrow \beta \equiv 0 .
$$

Note that the set $\left\{u(., T, \mathrm{v}) ; \mathrm{v} \in \boldsymbol{L}^{2}(\Theta \times(0, T))\right\}$ is a closed subspace of $E$.

Let us consider $\varphi$ as being a solution of the ad- joint system

$$
\mid \begin{aligned}
& -\left(\varphi_{t}, e\right)+\mu a(\varphi, e)-\alpha((h \cdot \nabla) \varphi, e)+ \\
& \left.+a\left(\int_{t}^{T} g(\eta-t) \varphi, e\right)+(\nabla \pi, e)=0\right) \\
& \phi(x, T)=\beta \in E
\end{aligned}
$$

For all $e \in E$. System (20) has a unique solution $\varphi \in C^{0}([0, T] ; E)$.

Taking $e=u(t)$ in (20), using the lemma 3.1 (see Appendix) and observing that

$$
((h(t) . \nabla) \varphi(t), u(t))=-((h(t) . \nabla) u(t), \varphi(t)), \forall t \in[0, T],
$$

We get, after integration by parts in $t$, that

$$
\begin{aligned}
& -(u(T), \varphi(T))+\int_{0}^{T}\left[\left(u_{t}, \varphi\right)+\mu a(u, \varphi)\right] d t+ \\
& \left.\int_{0}^{T}[\alpha(h \cdot \nabla) u, \varphi)+a(g * u, \varphi)+(\nabla \pi, u)\right] d t=0
\end{aligned}
$$

By (18) we obtain

$$
-(u(T), \beta)=\int_{0}^{T}\left(\mathrm{v} \chi_{\Theta}, \varphi\right) d t
$$

Hence,

$$
0=\int_{0}^{T}\left(\mathrm{v} \chi_{\Theta}, \varphi\right) d t, \forall \mathrm{v} \in L^{2}(\Theta \times(0, T)
$$

So, one assures

$$
\varphi \equiv 0 \text { in } \Theta \times(0, T)
$$

Since $\varphi \equiv \sum_{t=1}^{N} \varphi i(t) e i$ and thanks to (10), we can guarantee by (24) that $\varphi i(t)=0$, for $i=1, \ldots, N$. Thus, 
$\varphi \equiv 0$ and, therefore $\beta \equiv 0$. Thus, system (18) is exactly controllable.

Step 2 (Estimates). Thanks to the results obtained in Step 1 , one can define the functional $M: L^{2}(0, T ; F) \rightarrow \mathbb{R}$ by

$$
M(h)=\inf _{\mathrm{v} \in U a d} \frac{1}{2} \int_{\Theta \times(0, T)}|\mathrm{v}|^{2} d x d t
$$

where $U_{a d}$ is the set of admissible controls $U_{a d}=\left\{\mathrm{v} \in \boldsymbol{L}^{2}(\Theta \times(0, T))\right\}, u$ solution of $\left.(18)\right\}$, satisfying

(13). Note that $U_{a d}$ is a closed convex of $\boldsymbol{L}^{2}(\Theta \times(0, T))$ and $J(\mathrm{v})$ is convex $s . c . i$ and coercive.

We are interested in proving that

$$
M(h) \leq\{\text { constant independent of } h \text { and } \alpha\}
$$

For this, we use a duality argument.

We consider the continuous linear operator $L: \boldsymbol{L}^{2}(\Theta \times(0, T)) \rightarrow F$ defined by $L(V)=u(., T ; V)$ and we introduce the functional.

$$
F_{1}(\mathrm{v})=\frac{1}{2} \int_{\odot \times(0, T)}|\mathrm{v}|^{2} d x d t
$$

And

$$
F_{2}(B)=\left\{\begin{array}{l}
0, \text { if } \beta=u^{T} \\
\infty, \text { otherwise }
\end{array}\right.
$$

In this way, we can rewrite the functional $M$ as follows:

$$
M(h)=\inf _{v \in \boldsymbol{L}^{2}(\vartheta \times(0, T))}\left[F_{1}(v)+F_{2}(L(v))\right]
$$

From the duality theorem of Frenchel and Rockefeller, see [29] we have.

$$
-M(h)=\inf _{\beta \in E}\left[F_{1}^{*}\left(L^{*}(\beta)+F_{2}^{*}(-\beta)\right]\right.
$$

where $L^{*} E \rightarrow \boldsymbol{L}^{2}(\Theta \times(0, T))$ is the adjoin of $L$.

Using (22), one sees that

$$
L^{*}(\beta)=-\varphi \text { in } \Theta \times(0, \mathrm{~T}) .
$$

Since

$$
F_{1}^{*}(\varphi)=\frac{1}{2} \int_{\Theta \times(0, T)}|\varphi|^{2} d x d t
$$

And

$$
F_{2}^{*}(-\beta)=-\left(\beta, u^{T}\right)
$$

Then

$$
-M(h)=\inf _{\beta \in E}\left[\frac{1}{2} \int_{\Theta \times(0, T)}|\varphi|^{2} d x d t-\left(\beta, u^{T}\right)\right]
$$

As $\mathrm{E}$ is a subspace of finite dimension, it follow that the three norms $|\beta|,\|\beta\|$ and $\left(\int_{\Theta}|\beta|^{2} d x\right)^{\frac{1}{2}}$ are equivalent on $E$, on that

$$
C|e|^{2} \geq \int_{\Theta}|e|^{2} d x \geq c\|e\|^{2}, \forall e \in E
$$

with $C$ and $C$ positive constants that only depend on $E$, where

$$
|e|^{2}=\int_{\Omega}|e|^{2} d x, \forall e \in E .
$$

Thus, (28) gives

$$
-M(h) \geq \inf _{\beta \in E}\left[\frac{c}{2} \int_{\odot \times(0, T)}|\varphi|^{2} d x d t-\left(\beta, u^{T}\right)\right]
$$

Now, we take $e=\phi(t)$ in (20) and integrating from $t$ to $T$. Then the terms containing $h$ drop out and we obtain

$$
\begin{aligned}
& \frac{1}{2}|\varphi(t)|^{2}+\int_{t}^{T} \mu a(\varphi, \varphi) d s+ \\
& \int_{t}^{T} a\left(\int_{t}^{T} g(\eta-t) \varphi, \varphi\right) d s=\frac{1}{2}|\beta|^{2} .
\end{aligned}
$$

Integrating in $(0, T)$ we get

$$
\begin{aligned}
& \frac{1}{2} \int_{0}^{T}|\varphi(t)|^{2} d t+\int_{0}^{T} t a(\varphi, \varphi) d t+ \\
& \int_{0}^{T} t a\left(\int_{0}^{T} g(\eta-t) \varphi, \varphi\right) d s=\frac{T}{2}|\beta|^{2}
\end{aligned}
$$

Notice

that

$$
a(\varphi, \varphi)=\|\varphi\|^{2} \leq C|\varphi|^{2}
$$

and

$a\left(\int_{t}^{T} g(\eta-t) \varphi, \varphi\right) \leq\|g\|_{\infty}\|\varphi\|^{2}$ for some $C>0$ that depends only on $E$ because $E$ are finite dimensional.

So

$$
\frac{T}{2}|\beta|^{2} \leq\left(\frac{1}{2}+C T+T\|g\|_{\infty}\right) \int_{0}^{T}\|\varphi(t)\|^{2}
$$

For suitable $C>0$ that depends only on $E$. In view of (31) we get

$$
-M(h) \geq \inf _{\beta \in E}\left[k\|\beta\|^{2}-\left(\beta, u^{T}\right)\right]
$$

where

$$
k=\frac{c T}{2+4 T+4 T\|g\|_{\infty}} \text { Hence, }
$$

Which implies (26).

Step 3 (Nonlinear system) Let $h \in L^{2}(0, T ; E)$ be given. We choose for $\mathrm{v}$ the unique element such that

$$
\frac{1}{2} \int_{\Theta \times(0, T)}|v|^{2} d x d t=M(h) \text {. }
$$

We define a continuous mapping $h \rightarrow v$ from $L^{2}(0, T ; E)$ in $\boldsymbol{L}^{2}(\Theta \times(0, T))$. We denote by $u(h)$ the solution of (18) with the control $v=u(h)$. Taking $\mathrm{e}=u(t)$ in (18) we obtain.

$$
\begin{aligned}
& \frac{1}{2} \frac{d}{d t}\|u(t)\|^{2}+\mu a(u(t), u(t))+ \\
& +a\left((g * u)(t), u(t)=\left(v \chi_{\Theta}, u(t)\right) .\right.
\end{aligned}
$$


Thus, integrating the last equation in $(0, t)$ we obtain

$\frac{1}{2}\|u(t)\|^{2}+\int_{0}^{t} \mu a(u(s), u(s)) d s+\int_{0}^{t}(a(g * u)(s), u(s)) d s$

$\leq\|v\|_{\mathbf{L}^{2}(\mathscr{O} \times(0, T))}\|u\| u(h)_{\mathbf{L}^{2}(\mathscr{O} \times(0, T))}$

By lemmas 3.1 and 3.2 we have

$$
\|u(t)\|^{2} \leq\|v\|_{\mathbf{L}^{2}(\Theta \times(0, T))}^{2}\|u\|_{\mathbf{L}^{2}(\Theta \times(0, T))}^{2}
$$

From Gronwall's inequality, we have by (38) that

$$
\|u(t)\|^{2} \leq C T\|v\|_{L^{2}(\varrho \times(0, T))}^{2}
$$

In view of (26) we obtain that, when $h$ varies in $L^{2}(0, T ; E)$

$$
u \text { remains a bounded subset } K_{1} \subset L^{2}(0, T ; E) \text {. }
$$

We claim that

the map $h \rightarrow u(h)$ admits a fixed point in $K_{1}$.

In fact, according to Schauder's fixed point theorem, it suffices to show that the range of $\mathrm{u}(h)$ when $h$ spans $K 1$ is relatively compact in $K 1$, which follows from the following estimate:

$$
\begin{aligned}
& u_{t} \text { remains in a bounded } \operatorname{set} L^{2}(0, T ; E) \\
& \text { when h describes } K_{1} .
\end{aligned}
$$

To prove (42) we observe that, from (18), the following holds

$$
\begin{aligned}
& \left|\left(u_{t}, e\right)\right| \leq C(\alpha\|h(t)\|\|\nabla u(t)\|+\mu\|\nabla u(t)\|)\|e\| \\
& +C\left(\|\mathrm{v}\|_{L^{2}(\vartheta)}+\|g\|_{\infty}\|\nabla u(t)\|\right)\|e\|, \forall e \in E
\end{aligned}
$$

Since on the finite-dimensional space $E$ all the norms are equivalent. Therefore

$$
\begin{aligned}
& \left\|u_{t}\right\| \leq C(\alpha\|h(t)\|\|\nabla u(t)\|+\mu\|\nabla u(t)\|)+ \\
& +C\left(\|\mathrm{v}\|_{L^{2}(\vartheta)}+\|g\|_{\infty}\|\nabla u(t)\|\right), \forall e \in E,
\end{aligned}
$$

Which implies 42.

\section{APPENDIX}

In this section we will show any technical lemmas necessary to show the existence and uniqueness of solutions.

Lemma 3.1. Let $\mathrm{g}:[0, \infty) \rightarrow[0, \infty)$ be a function of $L 1(0, \infty)$ and $\mathrm{y}, \xi \in L^{2}\left(; T ; L^{2}(\Omega)\right)$ Then

$$
\begin{aligned}
& \int_{\Omega \times(0, T)}\left[\int_{0}^{t} g(t-s) y(s) d s\right] \xi(t) d t= \\
& \int_{\Omega \times(0, T)}\left[\int_{0}^{t} g(\eta-s) \xi(s) d \eta\right] y(t) d t
\end{aligned}
$$

\section{Proof. Consider}

$\hat{y}=\left|\begin{array}{l}y \text { in }[0, T] \\ 0 \text { out side of }[0, T]\end{array} \quad \tilde{\varsigma}=\right| \begin{aligned} & \varsigma \text { in }[0, T] \\ & 0 \text { out side of }[0, T]\end{aligned}$

$\hat{g}=\mid \begin{aligned} & g(s) \text { if } s \geq 0 \\ & 0 \text { if } s<0\end{aligned} \quad$ and $\quad \widehat{g^{*} y}=\mid \begin{aligned} & g^{*} y \text { in }[0, T] \\ & 0 \text { out side } \text { of }[0, T] .\end{aligned}$

Thus $\quad \tilde{y} \in L^{2}\left(\mathbb{R} ; L^{2}(\Omega)\right), \quad \tilde{\varsigma} \in L^{2}\left(\mathbb{R} ; L^{2}(\Omega)\right) \quad$ and $\mathrm{g}^{\tilde{g} \in L^{1}(\mathbb{R})}$ By Lemma 2.1 it follows that $g * y \in L^{2}(\mathbb{R} ; H)$ Therefore,

$$
\begin{aligned}
& \int_{\Omega \times(0 ; T)} \int_{0}^{t}[g(t-s) y(s) d s] \varsigma(t) d t= \\
& \int_{\Omega \times(0 ; T)} g^{*} y(t) \varsigma(t) d t= \\
& \int_{\Omega \times I R} \bar{g} * \tilde{y}(t) \tilde{\varsigma}(t) d t= \\
& \int_{\Omega \times I R} \tilde{g} * \tilde{y}(t) \tilde{\varsigma}(t) d t:= \\
& \int_{\Omega \times I R} \tilde{y}(t) \tilde{g} * \tilde{\varsigma}(t) d t= \\
& \int_{\Omega \times I R}\left[\int_{I R}[\tilde{g}(t-\eta) \tilde{\varsigma}(\eta) d \eta] \tilde{y}(t) d t=\right. \\
& \int_{\Omega \times I R}\left[\int_{I R} \tilde{g}(\eta-t) \tilde{\varsigma}(\eta) d \eta\right] \tilde{y}(t) d t= \\
& \int_{\Omega \times(0, T)} \int_{t}^{T}[g(\eta-t) \varsigma(\eta) d(\eta)] y(t) d(t),
\end{aligned}
$$

where by $\breve{g}$ we denote $\widetilde{\widetilde{g}}(x)=\tilde{g}(-x)$.

Lemma 3.2. For an arbitrary $\alpha, T \in \mathbb{R}$ with $\alpha>0, T>0$ and $h \in L^{2}\left(0, T ; L^{2}(\Omega)\right)=L^{2}(\Omega)$ we have

$$
I:=\int_{\Omega \times(0, T)}\left[\int_{0}^{t} e^{-a(t-s)} h(x, s) d s\right] h(x, t) d t d x \geq 0
$$

Proof. From Lemma 3.1 we obtain

$$
I=\int_{\Omega \times(0, T)}\left[\int_{0}^{T} e^{-a(t-s)} h(x, \eta) d \eta\right] h(x, t) d t d x
$$

Hence,

$$
I=\frac{1}{2} \int_{\Omega \times(0, T)}\left[\int_{0}^{T} e^{-\alpha|t-\eta|_{I R}} h(x, \eta) d \eta\right] h(x, t) d t d x
$$

We note that form this identity the lemma is true for the case $\alpha=0$. On the other hand, for $\alpha=0$, let $h \times(x, t)=h(x, t)$ in $\Omega \times(0, T)$ and $h \times(x, t)=0$ outside of $\Omega \times(0, T)$. Then, from identity above it follows that

$$
I=\frac{1}{2} \int_{\mathbb{R}^{n} \times \mathbb{R}}\left[\int_{\mathbb{R}} e^{-\alpha|t-s|} h^{*}(x, \eta) d \eta\right] h^{*}(x, t) d t d x
$$

We make the change of variables $t-\eta=\xi$ to obtain

$$
I=\frac{1}{2} \int_{\mathbb{R}^{n} \times \mathbb{R}}\left[\int_{\mathbb{R}} e^{-\alpha|\xi|_{I R}} h^{*}(x, t-\xi) d \xi\right] h^{*}(x, t) d t d x
$$

The key idea to use the utilize Fourier Transform of $h \times(x$, t). In fact, 


$$
\begin{aligned}
& I=\frac{1}{2 \sqrt{2 \pi}} \int_{\mathbb{R}^{n} \times \mathbb{R}} h^{*}(x, t) \times \int_{\mathbb{R}} e^{-\left.\alpha|\xi|\right|_{I R}} \times \\
& \left\{\int_{\mathbb{R}^{n} \times \mathbb{R}} e^{i(x, t-\xi) \cdot(y, \eta)} \hat{h}^{*}(y, \eta) d \eta d y\right\} d t d x= \\
& \frac{1}{2 \sqrt{2 \pi}} \int_{\mathbb{R}^{n} \times \mathbb{R}} h^{*}(x, t) \times \int_{\mathbb{R}^{n} \times \mathbb{R}} e^{i(x, t) \cdot(y, \eta)} \hat{h}^{*}(y, \eta) \times \\
& \left\{\int_{\mathbb{R}} e^{-i \xi \eta} e^{-\alpha|\xi|} d \xi\right\} d \eta d y d t d x= \\
& \frac{1}{2 \sqrt{2 \pi}} \int_{\mathbb{R}^{n} \times \mathbb{R}} h^{*}(x, t) \\
& {\left[\int_{\mathbb{R}^{n} \times \mathbb{R}} e^{i(x, t) \cdot(y, \eta)} \hat{h} *(y, \eta) \widehat{e^{-\alpha|\eta|_{I R}}} d \eta d y\right] d t d x=} \\
& \frac{1}{2 \sqrt{2 \pi}} \int_{\mathbb{R}^{n} \times \mathbb{R}} \widehat{e^{-\alpha|\eta| \mathbb{R}}} \hat{h^{*}}(y, \eta) \\
& {\left[\int_{\mathbb{R}^{n} \times \mathbb{R}} e^{i(x, t) \cdot(y, \eta)} h^{*}(x, t) d t d x\right] d \eta d y=} \\
& \frac{1}{2 \sqrt{2 \pi}} \int_{\mathbb{R}^{n} \times \mathbb{R}} e^{-\alpha|\eta| \mathbb{R}} \hat{h} *(y, \eta)- \\
& {\left[\int_{\mathbb{R}^{n} \times \mathbb{R}} e^{i(x, t) \cdot(y, \eta)} \overline{h^{*}(x, t)} d t d x\right] d \eta d y=} \\
& \frac{1}{2 \sqrt{2 \pi}} \int_{\mathbb{R}^{n} \times \mathbb{R}} \overline{e^{-\alpha|\eta|_{\mathbb{R}}}} \hat{h} *(y, \eta) \times \\
& \overline{\left[\int_{\mathbb{R}^{n} \times \mathbb{R}} e^{i(x, t) \cdot(y, \eta)} h^{*}(x, t) d t d x\right]} d \eta d y= \\
& \frac{1}{2 \sqrt{2 \pi}} \int_{\mathbb{R}^{n} \times \mathbb{R}} e^{-\alpha|\eta|_{\mathbb{R}}}|\overline{\hat{h}} *(y, \eta)|_{\mathbb{R}}^{2} d \eta d y . \\
& \frac{1}{2 \sqrt{2 \pi}} \int_{\mathbb{R}^{n} \times \mathbb{R}} e^{-\alpha|\eta|_{\mathbb{R}}}|\overline{\hat{h}} *(y, \eta)|_{\mathbb{R}}^{2} d \eta d y .
\end{aligned}
$$

Therefore

$$
I=\frac{1}{2 \sqrt{2 \pi}} \int_{\mathbb{R}^{n} \times \mathbb{R}} e^{-\alpha|\eta|_{\mathbb{R}}}|\overline{\hat{h}} *(y, \eta)|_{\mathbb{R}}^{2} d \eta d y .
$$

Recalling the well know formula

$$
\widehat{e^{-\alpha|\eta| \mathbb{R}}}=\frac{2 \alpha}{\sqrt{2 \pi}\left(\alpha^{2}+\eta^{2}\right)}
$$

We have that

$$
I=\frac{\alpha}{2 \pi} \int_{\mathbb{R}^{n} \times \mathbb{R}}\left(a^{2}+\eta^{2}\right)^{-1}|\hat{\bar{h}} * \times(y, \eta)|_{\mathbb{R}}^{2} d \eta d y \geq 0
$$

where by $h *$ and $h *$ we denote the Fourier Transform and the conjugate of $h *$ respectively. This closes the proof of the Lemma.

\section{REFERENCES}

[1] J. Serrin, "Mathematical principles of clas- sical fluid mechanics," Handbuch der Physik, vol. 8, Springer-Verlag, 1959, pp. 125-263.
[2] J. C. Maxwell, "On the dynamical the- ory of gases, Philos," Trans. Roy. Soc., London, vol. 157, 1868, no. 35, pp. 49-88.

[3] J. C. Maxwell, "On the dynamical the- ory of gases," Philos. Magazine, pp. $129-145,1859$

[4] K. W. Thomson, "On the theory viscoelas- tic fluids," Math. A. Phys., 1875 , vol. 3, pp. 27-84.

[5] W. Voigt, "Uber die innere Reinbung der fes- ten Korper," Insbessondere Der Krystalle, Abh. Konigl. Ges. Wiss. Gottingen, vol. 36,1889 , no. 1 .

[6] W. Voigt, "Uber innere fester Korper, ins- bessondere der Metalle," Ann. Phys., vol. 283, 1892, pp. 671-693.

[7] J. G. Oldroyd, "Non-newtonian flow of liquids and solids," Rheology: Theory and Applica- tions., vol. 1, 1959, pp. 653-682.

[8] J. G. Oldroyd, Non-Linear Stress, Rate of Strain Relations at Finite Rates of Shear in So-Called Lin- Ear Elastico-Viscous Liquids, Jerusalem Aca- demic Press, Jerusalem, and Pergamon Press, Oxford, 1964 , pp. 520-529.

[9] G. Astarita and G. Marruci, Principles of non-Newtonian fluid mechanics, McGraw-Hill, 1976.

[10] W. L. Wilkinson, Non-Newtonian Fluids, Perg- Amon Press, Oxford, 1960.

[11] A. P. Oskolkov, "Initial boundary value problems for the equations of motion of Kelvin- Voigt fluids and Oldroyd fluids," in Proc. the Steklov, Institute of Mathematics, 1989, no. 2.

[12] A. P. Oskolkov and M. M. Akhmatov, "Convergent difference schemes for equations of motion of oldroyd fluids," J. Sov. Math. 2, no. 4, 1974.

[13] A. P. Oskolkov and A. A. Kotsiolis, "Solvabil ity of the basic initial boundary problem for the equations of motion of an Oldroyd fluid on $(0, \infty)$ and the behavior of its solutions as $\mathrm{t} \rightarrow \infty$, , lnst. V. A. Steklova AN SSSR, vol. 150, pp. 48-52, 1986.

[14] A. P. Oskolkov, N. A. Karazeeva and A. A. Kotsiolis, "Dynamical system generated by the equations of motion of an Oldroyd fluid of order L," lnst. V. A. Steklova AN SSSR, vol. 164, pp. 47-53, 1987.

[15] R. Temam, "Navier-stokes equations," Lecture Notes in Mathematics, vol. 2, 1979.

[16] A. V. Fursikov and O. Y. Imanuvilov, "Control- lability of evolution equations," Lecture Notes Series, Seoul National University Research Institute of Mathematics Global Analysis Center, Seoul, vol. 34, 1996.

[17] O. Y. Imanuvilov, "Remarks on exact controllability for the Navier stokes equations," ESAIM Control Optim. Calc. Var., vol. 6, 2001, pp. 39-72.

[18] E. F. Cara, S. Guerrero, O. Y. Imanuvilov, and J. P. Puel, "Local exact control- lability of the Navier-Stokes system," J. Math. Pures Appl., vol. 83 , no. 12,2004 , pp. 1501-1542.

[19] J. M. Coron, "On the controllability of the 2- D incompressible Navier-Stokes equations with the Navier slip boundary conditions," ESAlM Control Optim. Calc. Var., vol. 1, 1995, pp. 35-75.

[20] J. M. Coron and A. V. Fursikov, "Global exact controllability of the 2D Navier-Stokes equa- tions on a manifold without boundary," Russian J. Math. Phys., vol. 4, no. 4, 1996, pp. 429-448.

[21] J. L. Lions and E. Zuazua, "Contrôlabilité exacte des approximations de Galerkin des équations de Navier-Stokes," C. R. Acad. Sci. Paris, vol. 234, 1997, pp. 1015-1021.

[22] J. L. Lions and E. Zuazua, "Exact boundary controllability of GalerkinSs approximations of Navier-Stokes equations," Ann. Scuola Norm. Sup. Pisa Cl. Sci., no. 4, 1998, pp. 605-621.

[23] F. D. Araruna, F. W. C. Silva, and M. A. R. Medar, "Exact controllability of Galerkin's approximations of micropolar fluids," in Proc. the American Matheamtical Society, vol. 138, no. 4, April 2010, pp. 1361-1370.

[24] A. V. Fursikov, "Optimal control of distributed systems," Theory and Applications, American Mathematical Society, Providence, vol. 187, 2000.

[25] A. V. Fursikov, M. Gunzburger and L. Hou, "Optimal boundary control for the evolutionary Navier-Stokes system: the three-dimensional case," SlAM J. Control Optim., vol. 43, no. 6, 2005, pp. 2191-2232.

[26] A. V. Fursikov, M. Gunzburger, and L. Hou, "Optimal dirichlet control and inhomogeneous boundary value problems for the unsteady Navier-Stokes equations," ESAlM, vol. 4, 1998, pp. 97-116.

[27] E. F. Cara and S. Guerrero, "Local exact controllability of micropolar fluids," J. Math. Fluid Mech., vol. 9, 2007, pp. 419-453.

[28] J. L. Lions, "Contrôlabilité exacte, perturba- tions et stabilisation de systémes distributes," Contrôlabilité Exacte, Rech. Math. Appl., Masson, Paris, vol. 8, 1988.

[29] R. T. Rockafellar, "Duality and stability in extremum problems involving convex func- tions," Pacific Joumal Mathematic, vol. 21, 1967, pp. 167-187. 


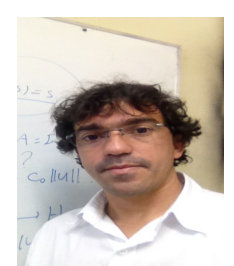

projects.

He is also the author of the following books: Advanced Calculus (2012) published by EDUEPB (2010), and published various research papers in the area of partial differential equations in various international journals.

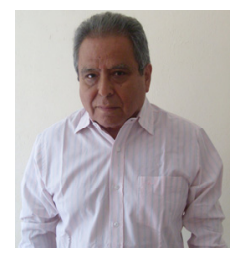

Manuel Antolino Milla Miranda was born in Lima, Cusco in 1941. After graduating with a $\mathrm{PhD}$ in mathematics in 1976 at Federal University of Rio de Janeiro, UFRJ, Brazil, he joined the University of Pierre et Marie Curie in France for a 2 year long postdoc position. He then transferred for another two years to Centre de Mathématiques Appliquées, École Polytechnique in France. From 1976 - 1994, he held a titular professor ship at Federal University of Rio de Janeiro, UFRJ, Brazil. In 1994, he became a full professor at Federal University of Fluminense, UFF, Brazil and transitioned back to Federal University of Rio de Janeiro, UFRJ, and Brazil where he is a full professor since 1997. In 2010, he accepted a Visiting Professor position at UEPB.
Manuel Antolino Milla Miranda is the author of the following books: Spectral Analysis in Hilbert Spaces(1993), An introduction to Sobolev Spaces and to Partial Differential Equations(1991), Sobolev Spaces (elliptical problems Initiation) published by editor IM-UFRJ(2000) and Introduction to Exact Control Theory. Method HUM published by EDUEPB(2014) and published various research papers in the area of partial differential equations in various international journals.

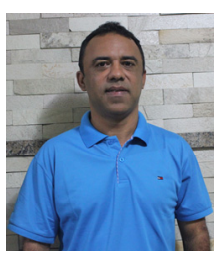

Aldo Trajano Lourêdo was born in Campina Grande, Paraíba in 1970. In 2008, he received his Ph.D. at Federal University of Rio de Janeiro, UFRJ, and Brazil in Mathematics. He joined as an adjoin professor on the Mathematics Department of UEPB in 2004 .

During the last decade, he was involved as a principal investigator in the following research projects

He is also the author of the following books: Advanced Calculus (2012) and Introduction to Exact Control Theory. Method HUM published by EDUEPB (2014), and published various research papers in the area of partial differential equations in various international journals. From 06/2009-06/2011, Aldo Trajano Lourêdo was elected as dean of the Mathematics Department at UEPB. 